\title{
RECONOCIMIENTO DE ACTIVIDADES EN EL DESPLAZAMIENTO POR INTERIORES MEDIANTE UN TELÉFONO INTELIGENTE Y SEÑALES DE OPORTUNIDAD
}

\author{
Fernando Seco y Antonio R. Jiménez \\ Centro de Automática y Robótica, CSIC-UPM, Arganda del Rey, Madrid \\ E-mail de contacto: fernando.seco@csic.es
}

\section{Resumen}

En este trabajo se estudia cómo las señales de oportunidad pueden ser utilizadas no sólo para mejorar la precisión del posicionamiento en interiores, sino también para obtener información valiosa de las actividades que está realizando el usuario. Las señales de oportunidad son aquellas no diseñadas especificamente para posicionamiento, pero presentes de forma natural en muchos entornos, y que pueden ser captadas, por ejemplo, a través de los sensores con los que cuenta cualquier teléfono móvil actual. Se introducen diferentes posibilidades de las señales de oportunidad, dentro de un marco de estimación de la posición bayesiano, y se ilustra su utilidad mediante trayectorias reales realizadas en un edificio de gran tamaño.

Palabras clave: Localización en interiores, señales de oportunidad, modelos bayesianos.

\section{INTRODUCCIÓN}

La localización de personas, robots o vehículos autónomos en entornos interiores es un problema de gran interés en la actualidad. Por analogía con el sistema de posicionamiento global (GPS), se habla de sistemas de posicionamiento locales (LPS) o de interiores (IPS). El enfoque habitual para conseguir la localización del individuo en un entorno de interiores consiste en la recogida de señales procedentes de un conjunto de balizas situadas en el edificio [SKRJ13], y la estimación de la posición mediante un algoritmo de tipo multilateración, filtro bayesiano o fingerprinting $\left[\mathrm{SJP}^{+} 09\right]$. Este esquema replica lo que es habitual en exteriores mediante GPS. Normalmente, no podemos confiar en disponer de una infraestructura dedicada para el posicionamiento en entornos interiores, sino que debemos emplear las señales naturalmente disponibles para mejorar nuestra estimación de la posición. En la literatura, estas señales reciben el nombre de señales de oportunidad [YNB14]. Así pues, un esquema más general para la determinación de la localización de un individuo en un entorno de interiores se ofrece en la figura 1.

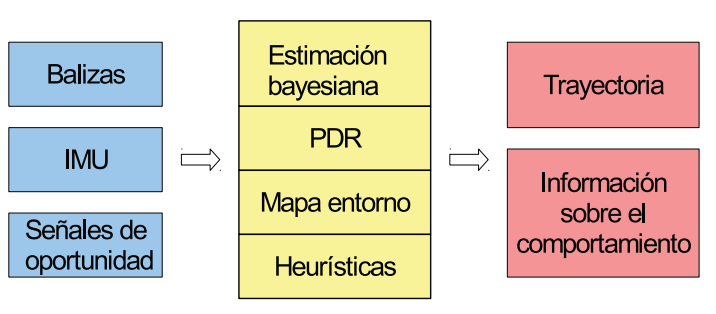

Figura 1: Diagrama general de bloques del procesamiento de señales para localización en entornos interiores.

En el caso de sistemas orientados a localización personal, las señales disponibles pueden ser obtenidas mediante un teléfono móvil: señales de balizamiento (sea por ejemplo puntos de acceso wifi, u otras más específicas como RFID, UWB, etc), señales procedentes del sensor inercial (IMU) contenido en el teléfono (proporcionando medidas de la aceleración, giro y campo magnético), y las señales de oportunidad, que dependen tanto del entorno como de las capacidades hardware del propio teléfono.

La información sensorial disponible es procesada por el segundo bloque, que incluye la metodología bayesiana (tal como filtro de Kalman, filtros de partículas, etc) [TBF05], el módulo PDR (Pedestrian Dead Reckoning, es decir, la odometría sobre los pasos detectados del usuario) [Har13], la información del plano del entorno (si está disponible), y finalmente diferentes heurísticas o procesamiento ad hoc que pueden aplicarse en determinadas circunstancias.

La metodología bayesiana de estimación se basa en una serie de principios [TBF05]: el primero es que el estado $x$ del móvil (persona, robot, etc) en un instante determinado $t$ está descrita por una función de densidad de probabilidad (PDF) $p\left(x_{t}\right)$. La evolución temporal de esta PDF (correspondiente al movimiento de la persona) se obtiene mediante medidas internas $u_{t}$, como las obtenidas mediante un odómetro, obteniéndose un modelo de movi- 
miento:

$$
p^{-}\left(x_{t}\right)=\int_{x} p\left(x_{t} \mid x_{t-1}, u_{t-1}\right) p\left(x_{t-1}\right) d x,
$$

siendo $u_{t-1}$ las señales recogidas por los sensores inerciales entre el instante $t-1$ y el instante $t$. En el caso de una persona caminando, el desplazamiento sólo puede estimarse cuando corresponde a pasos, por lo que los instantes discretos $t$ deben hacerse corresponder con los pasos detectados, como veremos en la sección 3.2.

La ecuación 1 plantea una hipótesis sobre la distribución de probabilidad $p\left(x_{t}\right)$ en el instante $t$ (de ahí el superdíndice - ). Las medidas $z_{t}$ recogidas en el instante $t$ nos permiten comprobar la bondad de nuestra estimación de la posición, mediante un modelo de medida que vincula las medidas sensoriales con la posición del usuario, siendo de la forma general:

$$
p^{+}\left(x_{t}\right)=p\left(z_{t} \mid x_{t}\right) p^{-}\left(x_{t}\right)
$$

Las ecuaciones 1 y 2 se aplican de forma consecutiva para realizar el seguimiento de la persona u objeto móvil. Para hacer tratable el problema, pueden plantearse simplificaciones sobre la distribución de probabilidad $p(x)$, que puede ser parametrizada como una distribución normal (filtro de Kalman), o tener forma arbitraria (usando filtros de partículas).

Como producto del procesamiento de la información recibida, puede obtenerse, además de la posición del usuario dentro de su entorno, cierta información cualitativa sobre su estado, intenciones o comportamiento. El propósito de este trabajo es demostrar que las señales de oportunidad recogidas mediante un teléfono móvil pueden mejorar la precisión del posicionamiento en interiores, y enriquecer la información de localización proporcionando indicios de algunos comportamientos del usuario.

\section{METODOLOGÍA EXPERIMENTAL}

Los datos empleados en este trabajo proceden de pruebas experimentales realizadas con motivo de una competición de sistemas de localización en el seno de la conferencia internacional Indoor Positioning and Indoor Navigation (IPIN 2016) organizada en la Universidad de Alcalá [20116]. La competición consiste en reconstruir una trayectoria desconocida seguida por un usuario en la escuela politécnica de la propia universidad, a partir de las señales recogidas por el teléfono, y unos datos previos de entrenamiento. Para hacer más realista la competición, el usuario ignora la posición de los

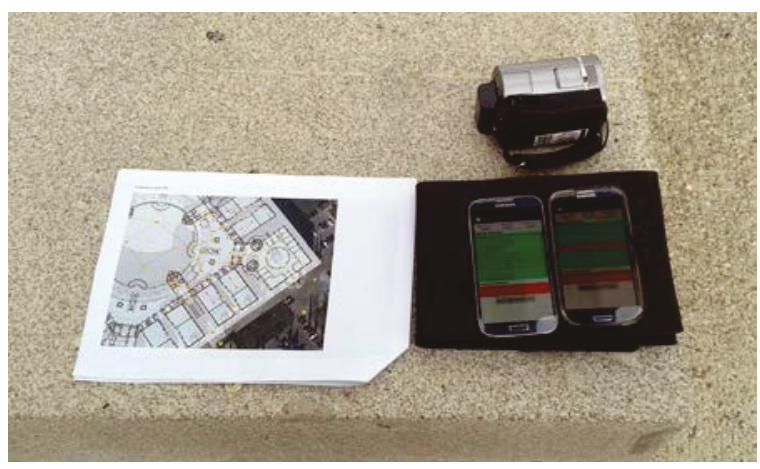

Figura 2: En los experimentos descritos en este trabajo, se recogieron las señales de oportunidad en varios recorridos por la Escuela Politécnica de la UAH mediante dos teléfonos móviles con un software especial. Además, las trayectorias se registraron en vídeo para referencia futura.

puntos de acceso wifi disponibles en el edificio y detectados por el teléfono.

Las pruebas consisten en unos recorridos por la escuela politécnica de la universidad y por nuestro edificio CAR-CSIC, donde el usuario porta dos teléfonos móviles, con una aplicación que recoge las diferentes señales disponibles desde el entorno (ver figura 2). Los recorridos, de aproximadamente 15 minutos de duración, se graban en vídeo para futura referencia y estudio de los diversos entornos por los que transcurre el desplazamiento. La extracción y procesamiento de las señales captadas por el teléfono se realizan en Matlab.

La tabla 1 muestra los diferentes sensores disponibles en un teléfono móvil convencional como los utilizados en los experimentos descritos en este trabajo y en la competición de IPIN 2016.

\section{USO DE LAS SEÑALES}

A continuación se ejemplifican diferentes usos de las señales recogidas por el móvil.

\subsection{Balizas de RF}

Las señales de uso más común para posicionamiento son las producidas por balizas de radiofrecuencia $(\mathrm{RF})$, normalmente wifi, aunque pueden emplearse otras tecnologías como bluetooth, RFID, UWB, etc. Como se muestra en la figura 3, existente una cantidad ingente de datos de fuerza de señal (RSS) e identidad (dirección MAC) de los diferentes nodos wifi recogidos por el teléfono. Existen varios enfoques para procesar esta información. Una posibilidad es la conversión de RSS a rango entre nodo wifi y teléfono mediante algún modelo teórico o experimental, y la triangulación 
Cuadro 1: Diferentes señales de oportunidad.

\begin{tabular}{|ll|l|}
\hline Sensor & Medida & Utilidad \\
\hline GNSS & Posición & Posición en entornos sin obstrucciones \\
Luz & Iluminancia & Detección de transiciones exterior / interior y puntos de luz \\
Wifi & Fuerza de señal RF & Detección de puntos de acceso inalámbricos \\
Micrófono & Intensidad sonora & Discriminación entre entornos \\
IMU & Aceleración, velocidad de giro & PDR y detección de movimiento \\
Barómetro & Presión atmosférica & Cambios de altura \\
Higrómetro & Grado de humedad & Cambios de habitación \\
\hline
\end{tabular}

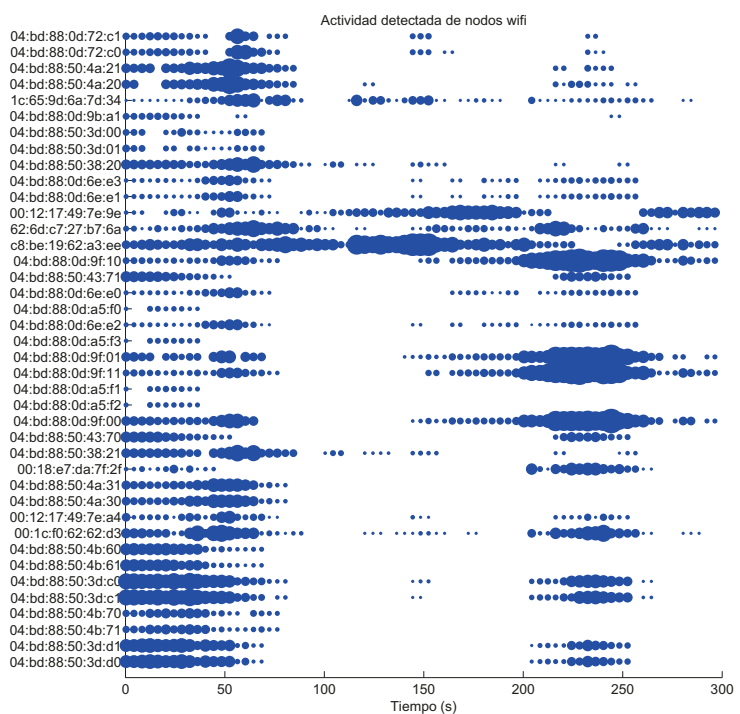

Figura 3: Actividad wifi recogida por un teléfono móvil durante 5 minutos en una trayectoria por la escuela politécnica de la UAH. El eje vertical contiene la dirección física (MAC) de cada punto de acceso wifi. Cada círculo corresponde a una detección por el móvil; el tamaño de dicho círculo es proporcional a la RSS. Sólo se muestran 40 de los 300 AP diferentes detectados.

para determinar la posición. Otra posibilidad es la incorporación de estas señales a un modelo de medida bayesiano del tipo $p(\operatorname{RSS} \mid x)$ (ver ecuación 2). En ambos casos, sería necesario conocer previamente la ubicación en el edificio de cada nodo wifi, lo cual es una labor costosa, aunque puede recurrirse a estrategias de tipo FastSLAM como las empleadas en Robótica [TBF05] como método de autocalibración de la posición de las balizas. Finalmente, son muy populares los métodos de aprendizaje automático o fingerprinting, para los cuales no es necesaria la determinación de la posición de las balizas.

\subsection{Odometría en interiores}

La odometría es la reconstrucción de la trayectoria seguida por el usuario a partir de señales relacio- nadas con su desplazamiento (en inglés, PDR o pedestrian dead reckoning). Dentro de la metodología bayesiana descrita en la introducción, corresponde al modelo de movimiento de la ecuación 1 , que, en nuestro caso, se expresa mediante una secuencia de valores $\left(l_{t}^{\mathrm{PDR}}, \delta \theta_{t}^{\mathrm{PDR}}\right)$, en los que se estima la longitud y el cambio de dirección espacial en cada paso.

Los métodos de odometría personal pueden proporcionar unas trayectorias relativamente fiables, con dos grandes desventajas: (a) la estimación es relativa, tanto en posición como en orientación, y (b) la deriva temporal hace que la trayectoria estimada se aparte mucho de la real en un tiempo relativamente corto. El primer problema se solventa con el acceso a señales que proporcionen información absoluta de la posición (como por ejemplo, GNSS, balizas wifi, o señales de oportunidad), que permiten reestimar la trayectoria en un marco de referencia absoluto. El segundo problema es el que supone una mayor limitación en el uso de las técnicas PDR. En el caso de sensores inerciales colocados en el pie, pueden emplearse los apoyos producidos en cada paso para compensar la deriva y la desviación sistemática de la trayectoria, técnica conocida como actualización de velocidad nula (o ZUPT por sus siglas en inglés) [SHNR10]. En los experimentos realizados por nuestro grupo con una IMU de calidad media (Xsens modelo MTi) colocada en el pie, el error relativo acumulado es del $1 \%$, o $1 \mathrm{~m}$ por cada $100 \mathrm{~m}$ recorridos, aproximadamente [JSPG10b]. Este error puede reducirse (y mantenerse acotado) mediante correcciones externas [JSPG10a].

En nuestro caso, las señales proporcionadas por la IMU contenida por el teléfono móvil son peores que las obtenidas en los trabajos citados debido a la menor calidad del acelerómetro y del giróscopo incluidos en el teléfono y a su ubicación no óptima (por no ir colocados en el pie, no puede emplearse algoritmos de actualización de velocidad nula). Por ese motivo, las trayectorias estimadas con PDR estarán sujetas a mayor deriva.

La figura 4 muestra una trayectoria recta de ida 


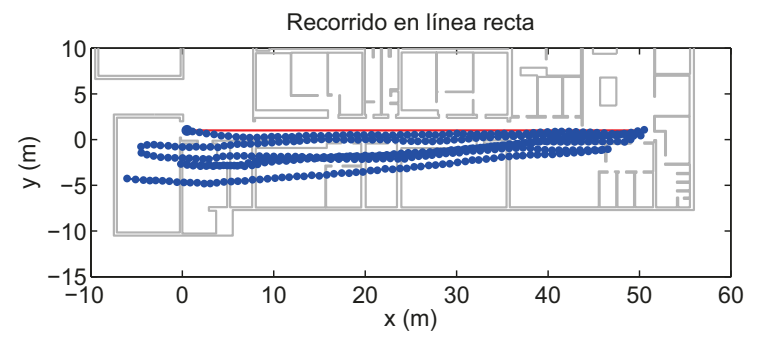

Figura 4: Trayectoria estimada por PDR, corrrespondiente a tres recorridos de ida y vuelta por un pasillo de $50 \mathrm{~m}$ de longitud.

y vuelta por un pasillo de $50 \mathrm{~m}$ de longitud, realizada varias veces de forma consecutiva. La longitud de paso se computa como proporcional al máximo valor absoluto de la variación de la aceleración, según el método descrito en [Wei02], el ángulo de giro en cada paso mediante las medidas del giróscopo y del magnetómetro. Como puede verse, los tramos rectos se estiman de forma razonable, aunque existe una incertidumbre en el ángulo estimado en cada giro al final del recorrido (que debería ser $180^{\circ}$ ). Este error se introduce como una deriva en la trayectoria; la discrepancia final es de $8 \mathrm{~m}$ (un $2.7 \%$ de la distancia total recorrida) respecto a la posición inicial.

El error es mucho mayor en trayectorias curvas o que no siguen un patrón determinado; tres ejemplos se muestran en la figura 5. La trayectoria (a) corresponde a un sector circular del edificio con un diámetro de $15 \mathrm{~m}$, como puede verse el giro se estima, pero de forma bastante incorrecta. En la parte (b) se recorren dos pasillos contiguos de despachos de profesores, unidos por un tramo circular. En ambos pasillos se estima incorrectamente el ángulo de retorno desde el fondo del pasillo a la parte principal del edificio. Finalmente, la parte (c) muestra la bajada por un tramo de escaleras; la posición $(x, y)$ final debería corresponder aproximadamente a la inicial.

Dos formas de solventar este error acumulado en las trayectorias estimadas con PDR en dispositivos móviles en la mano son: (a) el uso de planos del edificio; y (b) establecimiento de heurísticas sobre la trayectoria. En el primero, las paredes del edificio se contemplan en el modelo de desplazamiento (ecuación 1), introduciendo restricciones en los movimientos posibles y corrigiendo las derivas [LSVW11]. El segundo, se estudia el movimiento para detectar pautas en el mismo (tales como recorrer un pasillo en línea recta, bajar escaleras en círculo, etc), y las derivas se corrigen de forma ad hoc sobre la trayectoria esperada [BO10]. Ambas técnicas funcionan mejor en edificios con estructura regular, y en lugares relati- (a) Vuelta circular

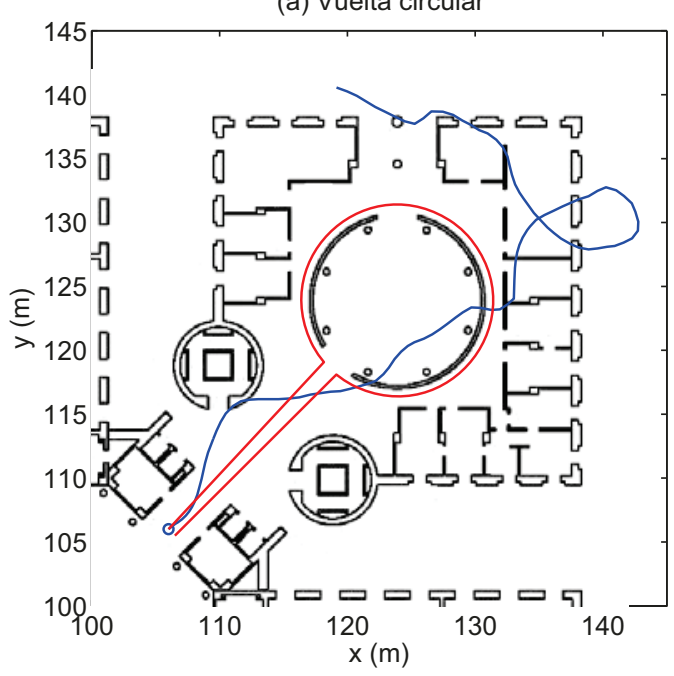

(b) Pasillos de profesores

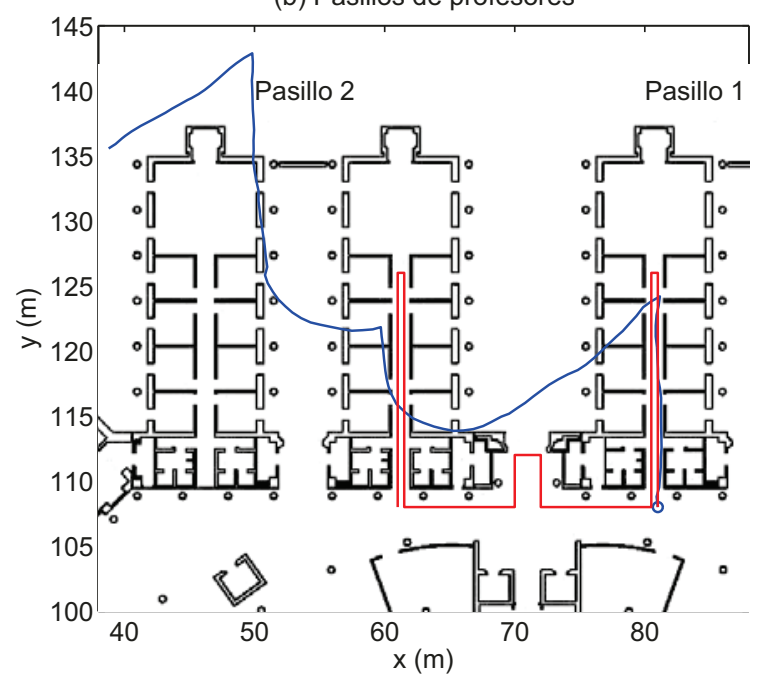

(c) Bajada por escaleras

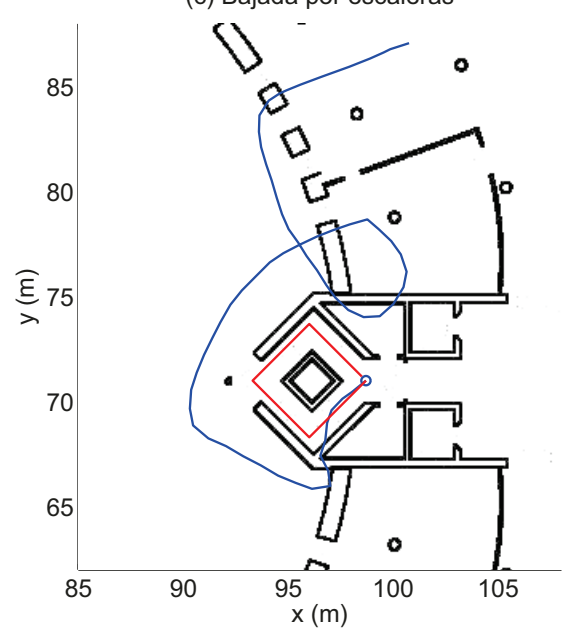

Figura 5: La deriva de los sensores inerciales afecta especialmente a la estimación de trayectorias con giros, como se muestra en estos ejemplos (en rojo, trayectorias reales, en azul, estimadas mediante PDR). 
vamente estrechos (como pasillos) y pierden exactitud en áreas del edificio diáfanas.

\subsection{Transición entre recintos interiores y exteriores}

Un aspecto importante de los sistemas de posicionamiento es la conmutación entre entornos exteriores e interiores. El dispositivo puede usar GPS en el exterior, y activar el sistema de interiores (IPS) al entrar en un edificio, idealmente de forma transparente para el usuario. Una forma de activar esta transición es emplear el sensor de iluminancia del que dispone un teléfono. En la figura 6 se muestra este efecto; se distingue perfectamente el acceso al edificio, el acceso al patio central ( $\sin$ techo) y la salida definitiva del edificio. En las zonas exteriores la luminancia varía entre 2000 y 18000 lux (correspondientes a un día nublado), mientras que en el interior del edifico los valores se mantienen por debajo de los 300 lux. Las variaciones en interiores (parte b) corresponden al paso de la persona por debajo de focos de luz existentes en el edificio. Esta variación puede emplearse para mejorar la localización bajo un método bayesiano de estimación de la posición [JZS14].

\subsection{Cambios de planta}

Los cambios de planta pueden detectarse de varias formas. Una de ellas es por el balizamiento de RF, ya que las señales de RF no suelen penetrar el forjado existente en el suelo entre una planta y otra; de esta forma, el conjunto de nodos wifi disponibles en una planta determinada no se detectarán en otra planta. Otra medida directa consiste en el cambio de presión barométrica con la altura.

Estos efectos se ilustran en la trayectoria de la figura 7, en la que una persona sube desde la planta baja a la tercera planta mediante el ascensor (minuto 10) y desciende de nuevo por las escaleras (minuto 16). Como puede observarse, el cambio de presión atmosférica producido (aproximadamente 2 milibares) es fácilmente detectable con el barómetro del teléfono móvil. Puede observarse además como esta variación es más lenta (0.022 mbar/s) bajando las escaleras a pie que subiendo en ascensor $(-0.083 \mathrm{mbar} / \mathrm{s})$. Otra forma de distinguir cómo se ha producido el desplazamiento vertical es tener en cuenta que el número de nodos wifi detectados es prácticamente nulo cuando estamos en el interior de la estructura metálica del ascensor.

Cabe destacar que la detección del movimiento de ascenso / descenso de los escalones únicamente mediante técnicas de navegación inercial (INS) resulta imposible en la práctica dada la gran deriva
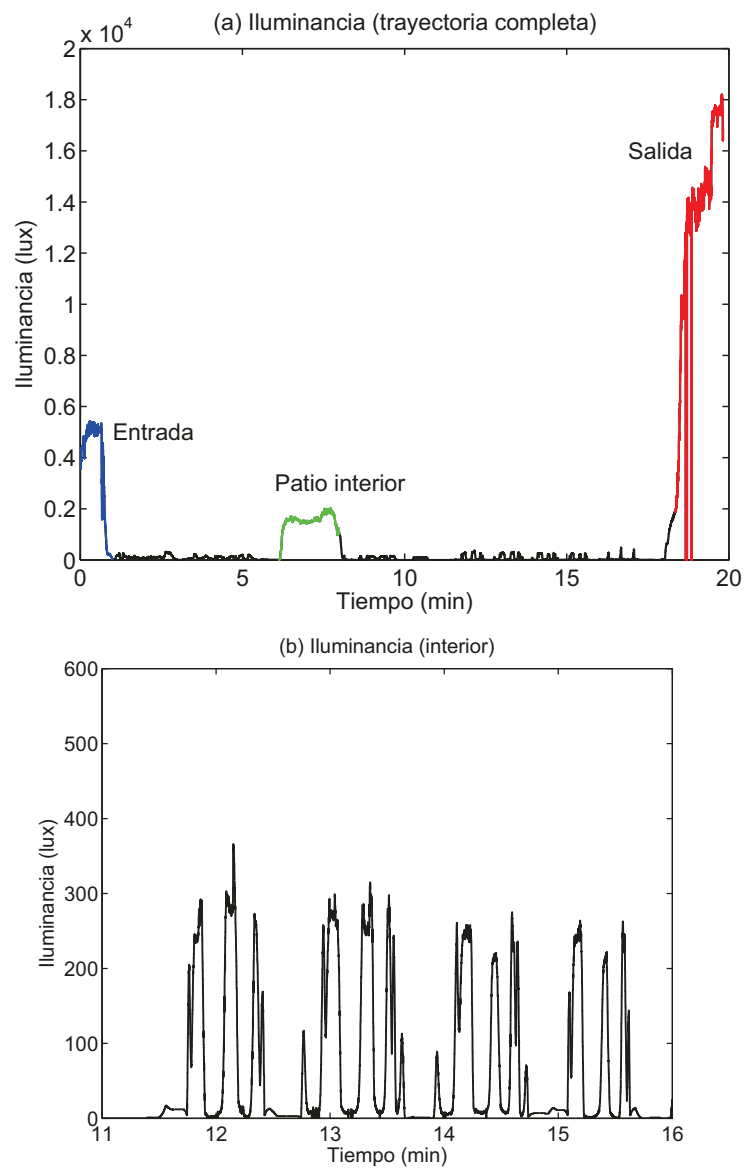

(c) Trayectoria exterior en el mapa

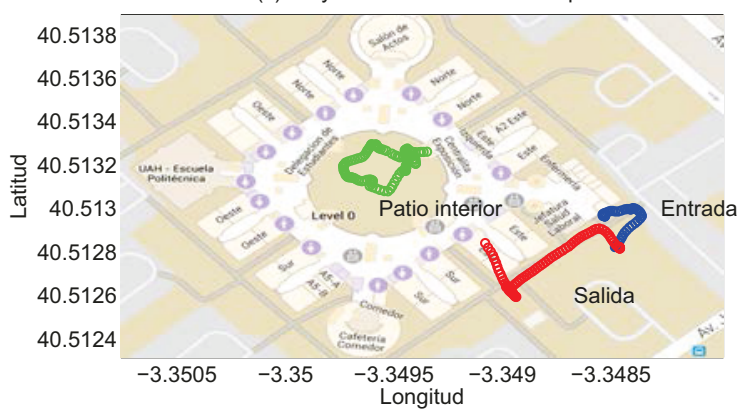

Figura 6: Variación de la iluminancia al pasar de la calle al interior del edificio (parte a). Los cambios de menor magnitud en el interior del edificio (parte b) corresponden al paso bajo fuentes de iluminación. En la parte c se muestra el mapa al que está referida la trayectoria. 
(a) Cambio de planta - variación de la presión atmosférica

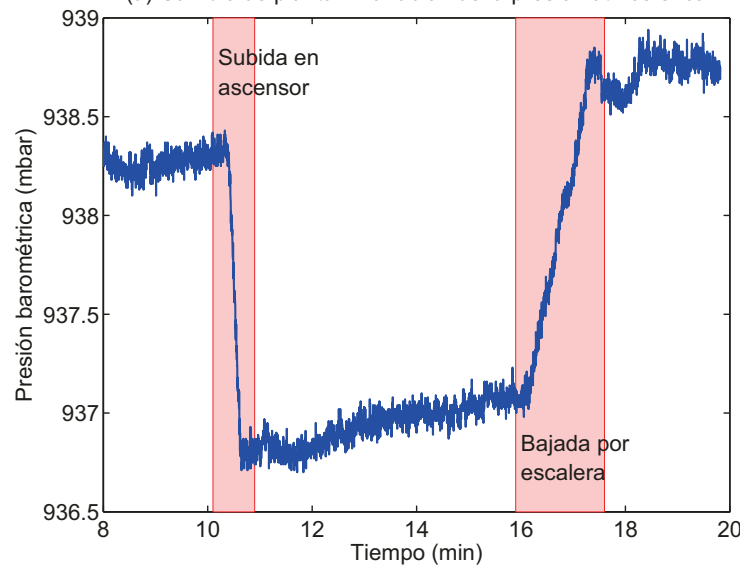

(b) Cambio de planta - detección APs wifi

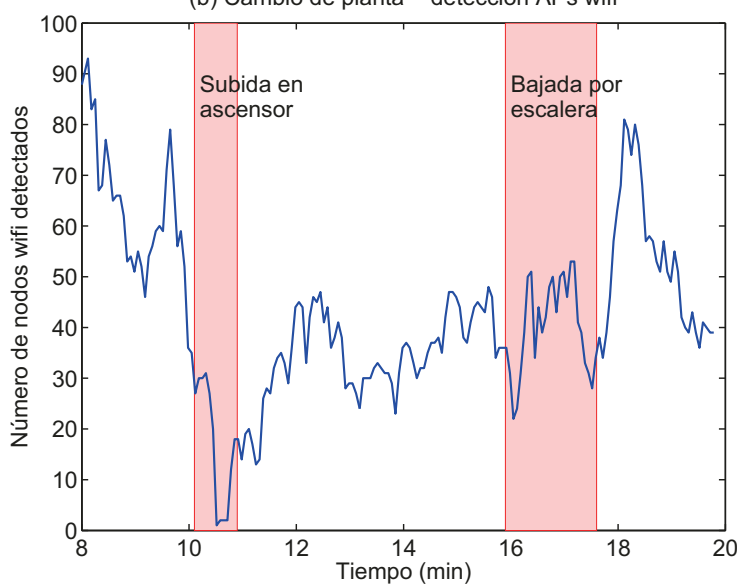

Figura 7: Variación de la presión atmosférica y de la actividad de detección de nodos wifi al cambiar entre plantas.

de los sensores inerciales presentes en un teléfono móvil. Con sensores de mayores prestaciones y una colocación óptima (en el pie), sí que resulta factible detectar el ascenso y descenso entre plantas.

\subsection{Temperatura y humedad}

Muchos teléfonos también disponen de sensores de temperatura y humedad, que proporcionan señales que pueden ser útiles para discriminar el lugar donde se encuentra el usuario. En los experimentos que realizamos medimos ambas variables en un recorrido que incluía cuatro lugares bien distintos: un laboratorio, un aseo, la cafetería y el taller mecánico (en cada uno de los cuales permanecimos 5 minutos), con los resultados mostrados en la figura 8. La primera conclusión que puede extraerse de la figura es que existen variaciones notables de temperatura y humedad que pueden emplearse para discriminar los cambios de habitación. Al igual que el caso de la presión atmosférica, deben emplearse las variaciones relativas de ambas magnitudes, ya que las medidas absolutas de los (a) Cambio de habitación - variación de la temperatura

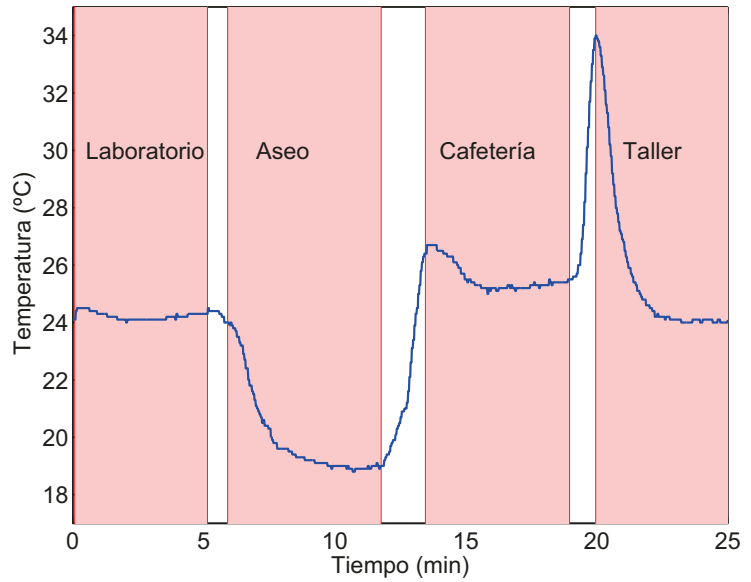

(b) Cambio de habitación - variación de la humedad

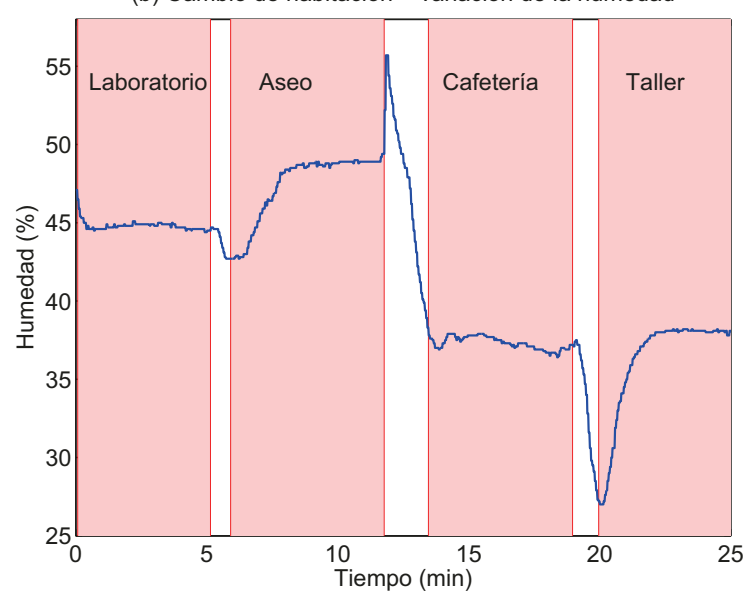

Figura 8: Variación de la temperatura y la humedad medidas por el teléfono en diferentes habitaciones de un edificio (zonas sombreadas). Los pasos entre el aseo y la cafetería y entre la cafetería y el taller discurren por el exterior.

sensores no son fiables, y varían día a día. También puede observarse que los sensores tienen un tiempo de respuesta relativamente lento (superior a 1 minuto), por lo que no son fiables para detectar transiciones rápidas, pero sí ofrecen resultados válidos transcurrido un tiempo de estabilización.

\subsection{Detección de acciones}

Finalmente las señales procedentes del sensor inercial (IMU) del teléfono móvil también pueden ser empleadas para detectar posibles acciones (voluntarias o involuntarias) desempeñadas por el usuario. Trabajos previos en la literatura muestran que la componente vertical de la aceleración proporciona información suficiente para discriminar entre varios tipos de movimientos $\left[\mathrm{BGS}^{+} 15\right]$. Así, en la figura 9 se muestra la aceleración vertical medida por el móvil para las siguientes acciones: (a) el usuario camina unos pocos pasos; (b) el usuario 
salta de forma vertical; (c) el usuario se deja caer al suelo (manteniendo asido el teléfono); (d) el usuario se sienta en una silla. Puede verse que en cada caso, tanto el patrón temporal como su análisis en frecuencia (figura 10) son distintos.

Las técnicas de discriminación de movimientos comienzan con una fase de entrenamiento, en la cual se extraen parámetros de las señales capturadas: por ejemplo, amplitud de la oscilación de la aceleración, estadísticas sobre los tiempos de cruce por cero de la señal temporal, distribución del espectro de frecuencias, autocorrelación de señales, etc. Una vez almacenados los parámetros numéricos, debe emplearse un clasificador de los habituales en métodos de aprendizaje automático: vecino más cercano, árboles de decisión, redes bayesianas, máquinas de soporte virtual, etc. La efectividad de estos métodos varía mucho entre personas, pero se han demostrado viables a la hora de discriminar acciones del usuario, dentro de un conjunto restringido de posibles acciones $\left[\mathrm{BGS}^{+} 15, \mathrm{NAO}^{+} 15\right]$.

\section{CONCLUSIONES Y TRABAJO FUTURO}

El objetivo último de las aplicaciones de posicionamiento de interiores es el guiado de personas, la asistencia en la consecución de sus objetivos, y la detección de posibles eventos peligrosos, como una caída. En este trabajo se ha estudiado cómo se puede mejorar la estimación de la posición y el seguimiento de trayectorias de usuarios en entornos de interiores mediante una combinación de (a) señales de balizamiento, (b) odometría personal, y (c) señales de oportunidad, recogidas mediante un teléfono móvil. En un entorno determinado, el estudio y modelado de las señales de oportunidad puede suplir las carencias de PDR (limitados por los sensores de bajas prestaciones existentes en teléfonos móviles) o la inexistencia de señales RF de posicionamiento propio. De esta forma, puede obtenerse mayor información del comportamiento del usuario del sistema, y mejorar la capacidad de ayuda de estos dispositivos.

\section{Agradecimientos}

Este trabajo ha sido financiado por los proyectos LORIS (MINECO ref. TIN2012-38080-C04) y TARSIUS (MINECO/FEDER ref. TIN201571564-C4-2-R).

\section{Referencias}

[20116] IPIN 2016. 7th international conference on indoor positioning and indoor navigation, Alcalá de Henares, Spain, october 4-7, 2016. http: //www3.uah . es/ipin2016/index.php, 2016.

$\left[\right.$ BGS $\left.^{+} 15\right]$ Dmytro Bobkov, Ferdinand Grimm, Eckehard Steinbach, Sebastian Hilsenbeck, and Georg Schroth. Activity recognition on handheld devices for pedestrian indoor navigation. In International Conference on Indoor Positioning and Indoor Navigation (IPIN), pages 1-10. IEEE, 2015.

[BO10] Johann Borenstein and Lauro Ojeda. Heuristic drift elimination for personnel tracking systems. Journal of $\mathrm{Na}$ vigation, 63(04):591-606, 2010.

[Har13] Robert Harle. A survey of indoor inertial positioning systems for pedestrians. IEEE Communications Surveys \& Tutorials, 15(3):1281-1293, 2013.

[JSPG10a] A. R. Jiménez, F. Seco, J. C. Prieto, and J. Guevara. Pedestrian indoor navigation by aiding a foot-mounted IMU with RFID signal strength measurements. In International Conference on Indoor Positioning and Indoor Navigation (IPIN), Zurich, Switzerland, 2010.

[JSPG10b] A.R. Jiménez, F. Seco, J. C. Prieto, and J. Guevara. Indoor pedestrian navigation using an INS/EKF framework for yaw drift reduction and a foot-mounted IMU. In WPNC: rth Workshop on Positioning, Navigation and Communication, 2010.

[JZS14] Antonio R Jiménez, Francisco Zampella, and Fernando Seco. Improving inertial pedestrian deadreckoning by detecting unmodified switched-on lamps in buildings. Sensors, 14(1):731-769, 2014.

[LSVW11] J. Á B. Link, P. Smith, N. Viol, and K. Wehrle. Footpath: Accurate mapbased indoor navigation using smartphones. In 2011 International Conference on Indoor Positioning and Indoor Navigation (IPIN), pages 1-8, Sept 2011.

$\left[\mathrm{NAO}^{+} 15\right]$ Phong Nguyen, Takayuki Akiyama, Hiroki Ohashi, Goh Nakahara, Katsuya Yamasaki, and Saito Hikaru. Userfriendly activity recognition using svm classifier and informative features. In International Conference on Indoor 


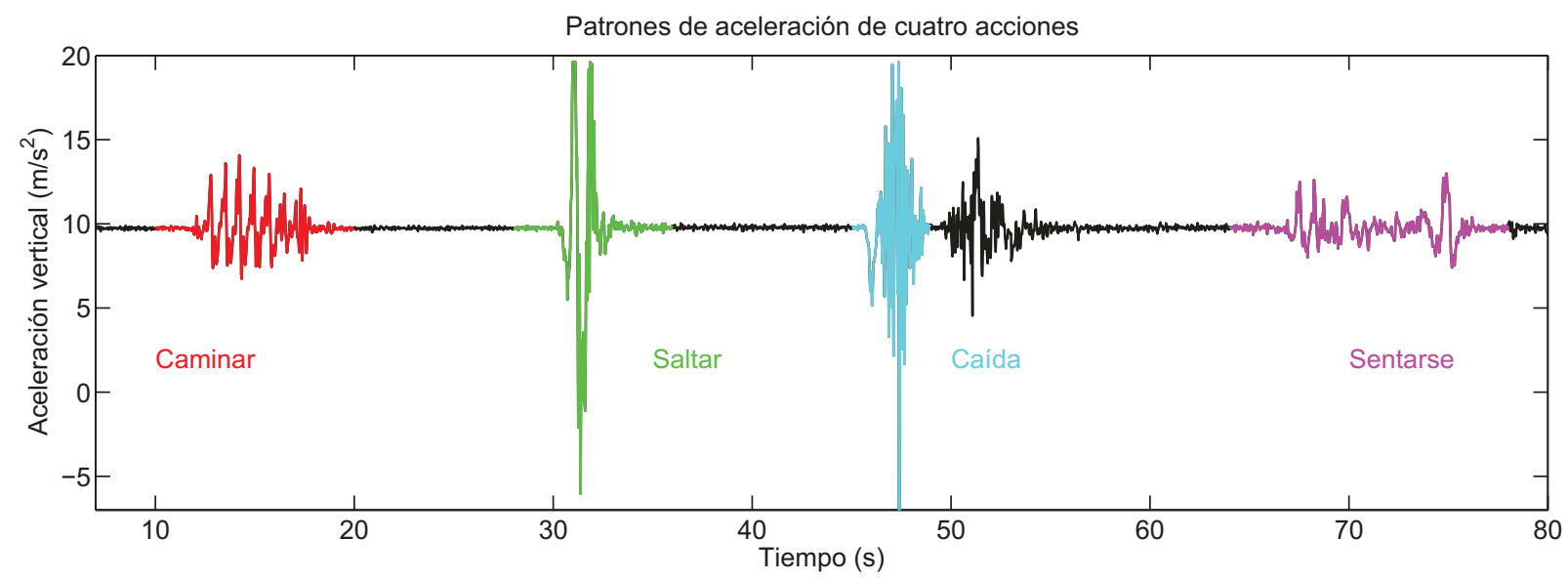

Figura 9: Patrón de aceleración vertical de cuatro acciones por un usuario: (a) caminar, (b) salto vertical, (c) caída al suelo, (d) sentarse en una silla. Las señales están muestreadas a $50 \mathrm{~Hz}$.
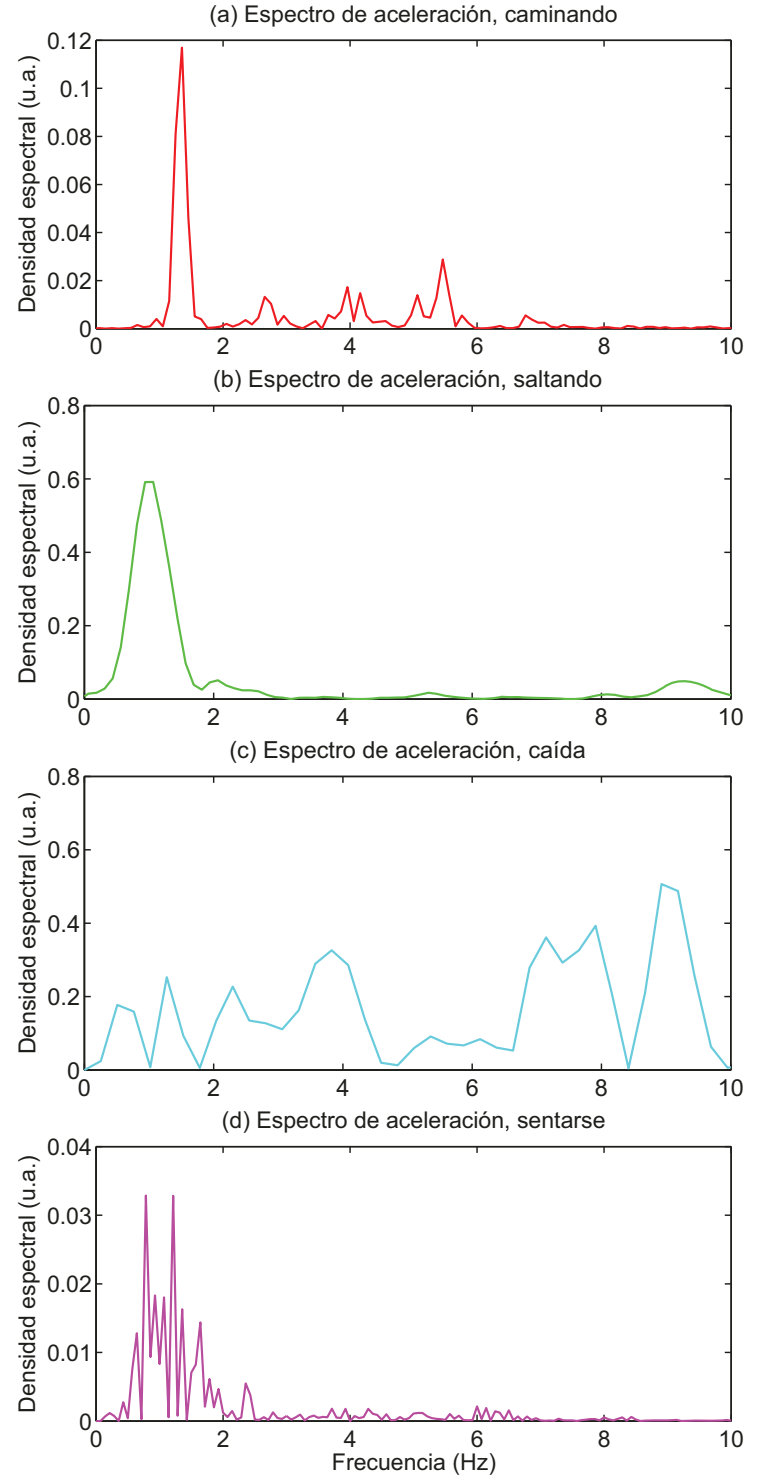

Figura 10: Densidades espectrales correspondientes a las señales de la figura 9 .
Positioning and Indoor Navigation (IPIN), pages 1-8. IEEE, 2015.

[SHNR10] I. Skog, P. Handel, J. O. Nilsson, and J. Rantakokko. Zero-velocity detection - an algorithm evaluation. IEEE Transactions on Biomedical Engineering, 57(11):2657-2666, Nov 2010.

$\left[\mathrm{SJP}^{+} 09\right]$ F. Seco, A.R. Jiménez, C. Prieto, J. Roa, and K. Koutsou. A survey of mathematical methods for indoor localization. In IEEE International Symposium on Intelligent Signal Processing (WISP), pages 9-14, aug. 2009.

[SKRJ13] Fernando Seco, Katerina Koutsou, Francisco Ramos, and Antonio R Jiménez. Localización personal en entornos interiores con tecnología RFID. Revista Iberoamericana de Automática e Informática Industrial RIAI, 10(3):313-324, 2013.

[TBF05] S. Thrun, W. Burgard, and D. Fox. Probabilistic Robotics. MIT Press, 2005.

[Wei02] Harvey Weinberg. Using the ADXL202 in pedometer and personal navigation applications. Analog Devices AN-602 application note, 2(2):1-6, 2002.

[YNB14] Chun Yang, Thao Nguyen, and Erik Blasch. Mobile positioning via fusion of mixed signals of opportunity. IEEE Aerospace and Electronic Systems Magazine, 29(4):34-46, 2014. 